\title{
Design Analysis of Thermoacoustic Refrigerator Using Air and Helium as Working Substances
}

\author{
B.G. Prashantha ${ }^{a, *}$, M.S. Govinde Gowda ${ }^{b}$, S. Seetharamu ${ }^{c}$, G.S.V.L. Narasimham ${ }^{d}$ \\ ${ }^{a}$ Department of Industrial Engineering \& Management, JSS Academy of Technical Education, Dr. Vishnuvardhana \\ Road, Bangalore-560 060, India \\ ${ }^{b}$ Department of Mechanical Engineering, Nagarjuna College of Engineering and Technology, Bengaluru-562 164, India \\ ${ }^{c}$ Central Power Research Institute, Bangalore-560 080, India \\ ${ }^{d}$ Department of Mechanical Engineering, Indian Institute of Science, Bangalore-560 012, India
}

\begin{abstract}
In this paper, thermoacoustic refrigerator design strategy with parameters normalization and literature review covering the recent development in the modification of the resonator shape and size is discussed. The design of a $10 \mathrm{~W}$ cooling power thermoacoustic refrigerator using air as working substance and the effect of operating frequency on viscous and thermal penetration depths, and on stack sheet thickness and spacing are discussed. The promising $10 \mathrm{~W}$ cooling power TDH (Taper and Divergent section with Hemispherical end) resonator design operating with air and helium gases as working substances is analyzed using DeltaEC software. The analysis results show that the TDH resonator design using helium as working substance operates at lower drive ratio (14\%) compared to air (25\%). In comparison, DeltaEC predicts a decent low temperature of $-35.4^{\circ} \mathrm{C}$ at cold heat exchanger with a COP of 0.5294 when operated using helium gas, and for air is $-9^{\circ} \mathrm{C}$ and 0.8113 respectively, and the results are discussed.
\end{abstract}

Keywords: Thermoacoustic, TDH design, Drive ratio, DeltaEC

\section{Introduction}

Since last three decades thermoacoustic refrigeration is considered as a new cooling technology. Thermoacoustic refrigerator is the most promising, eco-friendly, simple and alternative cooling device, which may replaces the existing predominant domestic vapour compression refrigeration (VCR) devices in the forthcoming years. Thermoacoustic refrigeration device makes use of thermoacoustic effect, which causes temperature gradient across the stack of sheets. Thermoacoustic effect occurs in the closed resonator tube filled with high pressure (about 10 bar) gases (air or inert gas like helium) because of sound waves generated by the loudspeaker. The cold and hot heat exchangers are placed on either side of the stack. The cold heat exchanger absorbs heat from the refrigerator chamber and then the stack pumps heat to the hot heat exchanger. The hot heat exchanger dissipates heat to the environment through the circulating cold water. This paper starts

${ }^{*}$ Corresponding author. Tel.: Tel.: + 9109731315226

Fax: + 9180 28612706; E-mail: bgpsandur@gmail.com

(C) 2016 International Association for Sharing Knowledge and Sustainability

DOI: $10.5383 /$ ijtee. 13.02 .006 with the various trends set by the researchers in resonator improvement during the last three decades. The strategy to be followed in designing a standing wave thermoacoustic refrigerator is discussed. The design of a $10 \mathrm{~W}$ cooling power thermoacoustic refrigerator using air as working substance is discussed and the effect of operating frequency on viscous and thermal penetration depths and on the stack sheet thickness and spacing are also discussed. An efficient TDH (Taper and Divergent section with Hemispherical end resonator) design found in the recent literature $[1,2]$ is validated using DeltaEC software operating with air and helium as working substances. The results are analyzed and the outcomes are discussed. Since air is free and abundantly available in the nature, an attempt is made in this paper to nullify the cost of the working substance using an efficient TDH resonator model. Therefore the objectives of this paper are to study the behaviour of the promising TDH resonator design using air as working substance and the results are compared with helium gas as working substance to obtain the concluding remarks. 


\section{Trends in resonator improvement}

In this section the various trends set by the researchers in the development of the resonator tube shape, size, and length to improve performance are discussed. The optimized resonator designs found in the literature till date are considered for the purpose of analysis. In the literature it is found that the resonator is optimized based on the minimum acoustic power loss as heat dissipation loss per unit surface area of the resonator system.

(a)

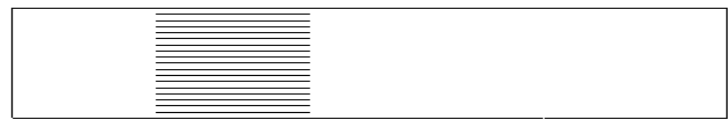

(b)

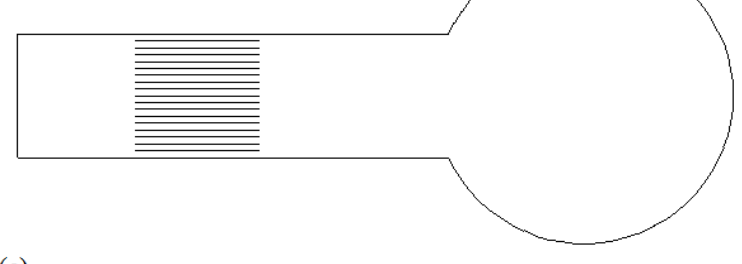

(c)

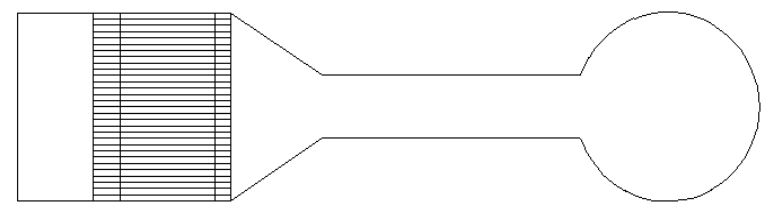

(d)

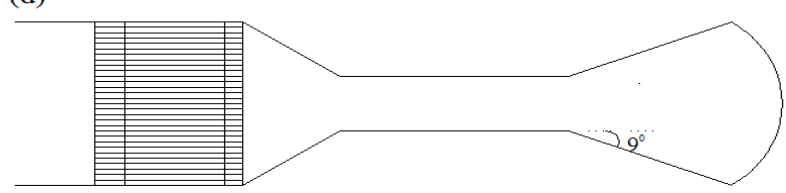

(e)

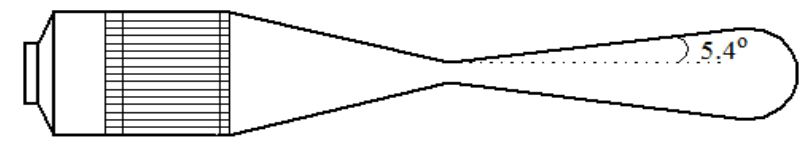

(f)

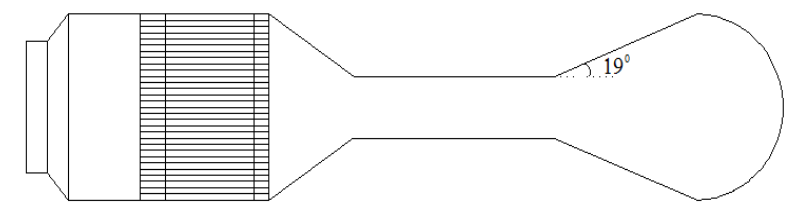

(g)

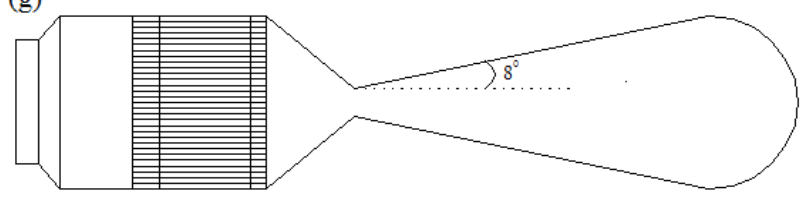

Fig.1. Resonator designs: a) Half-wavelength, b) Quarterwavelength with sphere buffer volume, c) Quarter-wavelength with two-diameter tube with sphere buffer volume, d) Quarterwavelength with two-diameter tube with conical buffer volume, e) Quarter-wavelength with CDH design, f) Quarter-wavelength with TSDH design, and g) Quarter-wavelength with TDH design.

The resonator tube should be strong enough to sustain high operating pressure (10 bar), and it should have compact structure without affecting performance. Therefore the resonator should have minimum possible length, diameter, surface area and volume leading to highest possible power density. The various resonator designs found in the literature are shown in Fig. 1 [112]. The first resonator design (Fig. 1 (a)) was the earlier halfwavelength resonator design having highest length, surface area, and volume. The second resonator design (Fig. 1 (b)) is the Hofler's quarter-wavelength resonator tube terminated with sphere buffer volume, which is further optimized by reducing diameter of the tube between the stack and sphere buffer volume (Fig. 1 (c)). Buffer volume of the Hofler's tube occupies much space and hence further improvement was made by Tijani et al. (Fig. 1 (d)) with two-diameter tube terminated with conical buffer volume. The first four resonator designs were having the cooling capacity less than or equal to $4 \mathrm{~W}$. The last three resonators were the recent theoretically optimized quarterwavelength $10 \mathrm{~W}$ cooling power resonator designs. The fifth resonator design (Fig. 1 (e)) is the $\mathrm{CDH}$ (Convergent-Divergent resonator terminated with Hemisphere) design. The theoretical evaluation of the $\mathrm{CDH}$ design is discussed elsewhere [6]. The sixth resonator design (Fig. 1 (f)) is the optimized TSDH (Taper, Small diameter tube and Divergent section with Hemispherical end resonator) design which is discussed elsewhere [1-3, 5-6]. The seventh design is the TDH (Taper and Divergent section with Hemispherical end resonator) design (Fig. $1(\mathrm{~g})$ ), is the theoretically optimized and efficient design compared to the designs as discussed above [1-2]. Therefore, in this paper an efficient TDH resonator design is chosen for the analysis using air and helium as working substances.

\section{Design Strategy of a Standing Wave Thermoacoustic Refrigerator}

In this section, the step by step design procedure to be followed in designing a laboratory scale thermoacoustic refrigerators models having the cooling capacity less than or equal to $10 \mathrm{~W}$ are discussed. The design steps discussed in this section may help the researcher to design and build the actual refrigerator models compatible for domestic cooling, replacing the conventional VCR units in the near future. The design strategy to be followed in designing individual components of a thermoacoustic refrigerator model is shown in Fig. 2. The design of thermoacoustic refrigerator begins with the first step 'design parameters identification'. The design parameters are identified under the four subgroups viz. design requirements parameters, operating parameters, geometry specific parameters, and material specific parameters. These parameters (about 25) are listed in Table 1. The design of thermoacoustic refrigerator involving 25 parameters is a difficult task and laborious in optimizing the design. Therefore, the parameters normalization technique is employed to reduce the number of parameters involved in the design to about 11 (Table 1). These normalized independent parameters are used in the design and optimization of the individual refrigerator components. The first component to start design is the stack. The stack is considered as the heart of the thermoacoustic refrigerator system, which is used for pumping heat from the cold heat exchanger to the hot heat exchanger. The performance optimization of the stack $\left(\mathrm{COP}_{\mathrm{s}}\right)$ is obtained using normalized cooling power and acoustic power equations [2-3, 6-7]. The stack length, center position, and acoustic power consumption are obtained using the normalized parameters equations (Table 1). The cross-sectional area of the stack and hence the diameter of the stack is derived using the normalized cooling power equation. The second component to design is the cold heat exchanger (CHX), which is placed at the right side of the stack in the quarter-wavelength resonator tube. 
The role of CHX is to absorb heat from the refrigerator cold chamber and transfer it to the oscillating gas surrounding the $\mathrm{CHX}$ in the resonator tube. The hot heat exchanger (HHX) placed on the other side of the stack is used for rejecting the heat pumped by the stack from CHX to the circulating cooling water. The diameter of the heat exchangers (HHX and CHX) is same as that of the stack. The length of the HHX should be about two times the length of CHX since it has to reject nearly twice the heat supplied by the CHX. In actual case the HHX has to reject the heat absorbed at $\mathrm{CHX}$ and the electrical power input to the driver (modified loudspeaker) [1-4, 6]. Similar to the stack, the length and acoustic power input to both the heat exchangers (HHX and CHX) is obtained using the normalized equations [2$3,6]$.

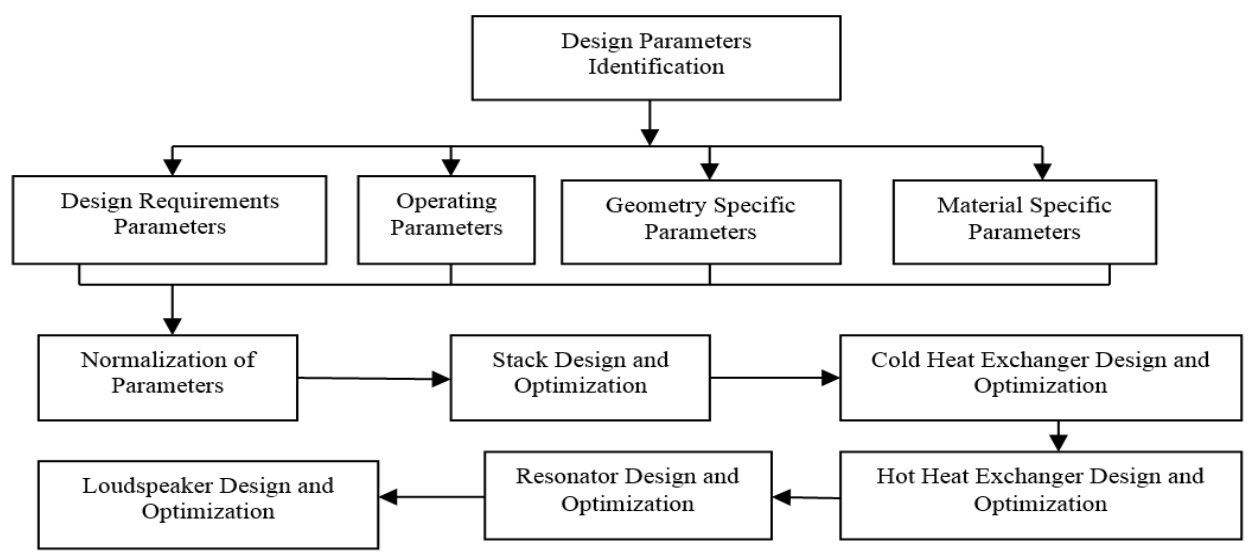

Fig. 2. The Design Procedure of Standing Wave Thermoacoustic Refrigerator.

Table 1. Thermoacoustic refrigerator design parameters and their normalized parameters.

\begin{tabular}{lc}
\hline 1. Design Requirements Parameters: & 5. Normalized Parameters Optimization \\
Cooling power $(Q)$ & Normalized stack length: \\
Desired temperature span $\left(\theta_{\mathrm{x}}\right)$ & $l_{3 \mathrm{n}}=k l_{3}$, \\
Total surface area of resonator $\left(A_{\mathrm{t}}\right)$ & Normalized stack center position: \\
Total resonator volume $\left(V_{\mathrm{t}}\right)$ & $X_{\mathrm{n}-\mathrm{s}}=k X$, \\
Power density of resonator system $\left(P_{\mathrm{v}}\right)$ & Normalized cooling power: \\
2. Operating Parameters: & $Q_{\mathrm{n}}=Q \div(P u A)$, \\
Resonator operating frequency $(f)$ & Normalized acoustic power: \\
Average gas pressure $(P)$ & $W_{\mathrm{n}}=W \div(P u A)$, \\
Dynamic pressure amplitude $\left(P_{\mathrm{a}}\right)$ & Drive-ratio: \\
Working gas & $D=P_{\mathrm{a}} \div P$, \\
Mean temperature of gas $\left(T_{\mathrm{mg}}\right)$ & Normalized temperature gradient: \\
Acoustic power input to resonator $(W)$ & $\theta_{n}=\theta_{\mathrm{x}} \div T_{\mathrm{mg},}$, \\
3. Geometry Specific Parameters: & Porosity or Blockage ratio: \\
Stack geometry & $\varepsilon=\mathrm{y} \div(\mathrm{y}+l)$, \\
Stack sheet spacing $(2 y)$ & Normalized thermal penetration depth: \\
Stack sheet thickness $(2 l)$ & $\delta_{\mathrm{kn}}=\delta_{\mathrm{k}} \div \mathrm{y}$. \\
Stack center position $(X)$ & Normalized viscous penetration depth: \\
Stack length $\left(l_{3}\right)$ & $\delta_{\mathrm{vn}}=\delta_{\mathrm{v}} \div \mathrm{y}$, \\
Stack cross-section $(A)$ & Prandtl number: $\sigma$, \\
Resonator geometry & Ratio of isobaric to isochoric specific heats: $\gamma$ \\
Resonator length $\left(L_{\mathrm{t}}\right)$ & \\
4. Material Specific Parameters: & \\
Stack material & \\
Stack density $\left(\rho_{\mathrm{s}}\right)$ & \\
Stack thermal conductivity $\left(K_{\mathrm{s}}\right)$ & \\
Stack specific heat $\left(C_{\mathrm{s}}\right)$ & \\
Resonator material & \\
Sound velocity $(u)$ & \\
Thermal conductivity of working gas $(K)$ & \\
Dynamic viscosity of working gas $(\mu)$ & \\
Specific heats of working gas $\left(C_{\mathrm{p}}, C_{\mathrm{v}}\right)$ & \\
Density of working gas $(\rho)$ & \\
Wave number $(k)$ & \\
Thermal and viscous penetration depths $\left(\delta_{\mathrm{k}}, \delta_{\mathrm{v}}\right)$ & \\
Thermal expansion coefficient of gas $(\beta)$ & \\
& \\
\hline
\end{tabular}


The fifth component to design is the quarter-wavelength resonator tube. The resonator tube accommodates stack-heat exchangers system (Fig. 1). The resonator tube should be perfectly insulated to avoid the loss of acoustic power input to the resonator system. The design and optimization of the stack, heat exchangers, and resonator tube is discussed elsewhere [1$3,5-9]$. The final component to design is the loudspeaker, which provides the necessory acoustic power input for the stack-heat exchanger system for heat pumping. The commercially available moving coil loudspeaker in the market has much less electro-acoustic efficiency of about 3-5\%. But the performance of the thermoacoustic refrigerator system mainly depends on the performance of the loudspeaker itself. Therefore the loudspeaker is modified with the back volume gas spring system called as driver is used for maximizing the electro-acoustic efficiency. The driver should operate at the operating frequency of the resonator tube for its efficient performance. The back volume gas spring system matches the driver frequency with the resonator frequency for maximizing the electro-acoustic efficiency of the driver. The driver has an electro-acoustic efficiency of about $45 \%$ as found in the literature [4]. The design and optimization of the loudspeaker is discussed elsewhere [4, 9].

\section{Design of a $10 \mathrm{~W}$ Thermoacoustic Refrigerator Using Air as Working Substance}

In this section, the design of a $10 \mathrm{~W}$ cooling power refrigerator using air as working substance for a temperature difference of $75 \mathrm{~K}$ across the stack is discussed. The scope of the design is limited to study the effect of operating frequency on the viscous and thermal penetration depths. And the effect of operating frequency on the stack sheet thickness and spacing as a function of thermal penetration depth are also discussed. The data used in the design calculations are given in Table 2.

Table 2. Data used in the design calculations

$$
\begin{aligned}
& Q=10 \mathrm{~W}, P=10 \text { bar, } \theta_{\mathrm{x}}=75 \mathrm{~K}, T_{\mathrm{hx}}=301 \mathrm{~K}, \\
& T_{\mathrm{mg}}=264 \mathrm{~K}, C_{\mathrm{pa}}=1.0282 \mathrm{kJkg}^{-1} \mathrm{~K}^{-1}, \gamma=1.39, \\
& u=324.8 \mathrm{~ms}^{-1}, K_{\mathrm{a}}=0.024 \mathrm{Wm}^{-1} \mathrm{~K}^{-1}, \\
& \mu_{\mathrm{a}}=1.6895 \times 10^{-5} \mathrm{kgm}^{-1} \mathrm{~S}^{-1}, \rho_{\mathrm{a}}=13.31 \mathrm{kgm}^{-3}, \\
& \sigma_{a}=0.724, \varepsilon=0.75
\end{aligned}
$$

It is chosen to operate the system at a mean temperature of air at $264 \mathrm{~K}$ across the stack. The guidelines in calculating the design parameters are discusses elsewhere [1-9]. The thermal and viscous penetration depths $\delta_{\mathrm{v}}$ and $\delta_{\mathrm{k}}$ are given by

$$
\begin{aligned}
& \delta_{k}=\sqrt{\frac{K_{a}}{\rho_{a} C_{\mathrm{pa}} \pi f}} \\
& \delta_{v}=\sqrt{\frac{\mu_{a}}{\rho_{a} \pi f}}
\end{aligned}
$$

and Prandtl number is given by

$$
\sigma_{\mathrm{a}}=\left(\frac{\delta_{\mathrm{v}}}{\delta_{\mathrm{k}}}\right)^{2}=\frac{\mu_{a} C_{\mathrm{pa}}}{K_{a}}
$$

Using the data given in Table 2 in Equations (1) and (2), thermal and viscous penetration depths as a function of operating frequency are calculated as given in Table 3. Calculation results show that the thermal and viscous penetration depths decreases with increase in operating frequency (Table 3). The porosity of the stack (blockage ratio) $\varepsilon$ is defined as

$\varepsilon=\frac{y}{(y+l)}$

where $l$ and $y$ are the half-stack plate thickness and spacing respectively.

Table 3. Viscous and thermal penetration depths as a function of operating frequency.

\begin{tabular}{ccc}
\hline $\boldsymbol{f}$ & $\tilde{\boldsymbol{o}}_{\mathrm{k}}$ & $\tilde{\boldsymbol{o}}_{\mathbf{v}}$ \\
$(\mathbf{H z})$ & $(\mathbf{m m})$ & $(\mathbf{m m})$ \\
200 & 1.6703 & 0.0494 \\
250 & 1.4940 & 0.0402 \\
300 & 1.3638 & 0.0367 \\
350 & 1.2627 & 0.0340 \\
400 & 1.1811 & 0.0318 \\
450 & 1.1136 & 0.0300 \\
500 & 1.0564 & 0.0284 \\
550 & 1.0072 & 0.0271 \\
600 & 0.9644 & 0.0259 \\
650 & 0.9265 & 0.0249 \\
700 & 0.8928 & 0.0240 \\
750 & 0.8626 & 0.0232 \\
800 & 0.8352 & 0.0225 \\
\hline
\end{tabular}

Using the data given in Table 2 in Equation (4), the stack thickness $2 l$ is calculated as a function of operating frequency $f$ at the various conditions of half-stack spacing expressed in terms of the thermal penetration depth $\delta_{\mathrm{k}}$. The calculation results are given in given in Table 4 . The stack spacing $(2 y)$ and thickness $(2 l)$ decreases with increase in operating frequency $f$. Also the stack spacing $(2 y)$ and thickness $(2 l)$ increases with increase in the value of halfstack spacing $(y)$ expressed in terms of the thermal penetration depth $\delta_{\mathrm{k}}$ (Table 4).

\section{Design Analysis of a 10 W Cooling Power TDH Resonator Design}

In section 2, the quarter-wavelength TDH resonator design is found to be an efficient design compared to the designs published elsewhere [1-6, 8-9]. In this section, an efficient quarter-wavelength $10 \mathrm{~W}$ cooling power TDH resonator design shown in Fig. 3 is used for the theoretical performance evaluation using air, and helium as working substances. The specifications of the TDH resonator design is given in Table 5 . The distance between the loudspeaker vibrating diaphragm and the hot heat exchanger $\left(l_{1}\right)$ is $28 \mathrm{~mm}$. The length of the hot heat exchanger $\left(l_{2}\right)$ is $14 \mathrm{~mm}$, the stack length $\left(l_{3}\right)$ is $75 \mathrm{~mm}$, and the cold heat exchanger length $\left(l_{4}\right)$ is $7 \mathrm{~mm}$. The length of the taper 
section $\left(l_{5}\right.$ or $\left.L_{2}\right)$ is $20 \mathrm{~mm}$. The length of the divergent section $\left(l_{6}\right)$ is $550 \mathrm{~mm}$. The radius of the hemisphere $R_{\mathrm{hs}}$ is $50 \mathrm{~mm}$. Therefore the total length of the resonator system is found to be $744 \mathrm{~mm}$. The stack and heat exchangers plates spacing $(2 y)$ and thickness $(2 l)$ are taken to be $1 \mathrm{~mm}$ and $0.557 \mathrm{~mm}$ respectively. At this condition the porosity $(\varepsilon)$ of stack-heat exchangers system is found to be 0.64 . The diameter of the stack $\left(d_{1}\right)$ is 3.6 $\mathrm{cm}$. Air is heavier than helium and hence the velocity of air is smaller than helium as shown in Table 2 [3]. Therefore to obtain better performance using air as a working substance, the diameter ratio $\left(d_{2} / d_{1}\right)$ at the throat section is taken to be 0.4 . A small diverging angle $\left(\theta_{2}\right)$ of $4.5^{\circ}$ is provided at the throat section to avoid eddies and turbulence in the divergent section. The hemisphere area $\left(A_{\mathrm{hs}}\right)$ and volume $\left(V_{\mathrm{hs}}\right)$ is found to be $0.01571 \mathrm{~m}^{2}$ and $2.618 \times 10^{-4} \mathrm{~m}^{3}$ respectively. At this TDH resonator geometry, the surface area $\left(A_{\mathrm{t}}\right)$, volume $\left(V_{\mathrm{t}}\right)$ and hence the power density $\left(P_{\mathrm{v}}\right)$ is found to be $1306.6 \mathrm{~cm}^{2}, 2.08 \mathrm{~L}, 4817.7$ $\mathrm{W} / \mathrm{m}^{3}$ respectively. The power density of the present TDH resonator design (Fig. 3) is in par with the optimized resonator designs published by the author of this paper [1-6].

Table 4. Stack sheet spacing and thickness against frequency as a function of thermal penetration depth.

\begin{tabular}{|c|c|c|c|c|c|c|c|c|c|c|}
\hline \multirow{4}{*}{$\underset{(\mathbf{H z})}{f}$} & \multicolumn{5}{|c|}{$\begin{array}{c}2 y \\
(\mathrm{~mm})\end{array}$} & \multicolumn{5}{|c|}{$\begin{array}{c}2 l \\
(\mathrm{~mm})\end{array}$} \\
\hline & $\mathbf{y}$ & $\mathbf{y}$ & $\mathbf{y}$ & $\mathbf{y}$ & $\mathbf{y}$ & $\mathbf{y}$ & $\mathbf{y}$ & $\mathbf{y}$ & $\mathbf{y}$ & $\mathbf{y}$ \\
\hline & $=$ & $=$ & $=$ & $=$ & $=$ & $=$ & $=$ & $=$ & $=$ & $=$ \\
\hline & $\tilde{\boldsymbol{o}}_{\mathbf{k}}$ & $1.25 \tilde{o}_{\mathrm{k}}$ & $1.5 \tilde{o}_{\mathrm{k}}$ & $1.75 \tilde{o}_{\mathrm{k}}$ & $2 \tilde{o}_{k}$ & $\tilde{\boldsymbol{o}}_{\mathbf{k}}$ & $1.25 \tilde{o}_{\mathrm{k}}$ & $1.5 \tilde{o}_{\mathbf{k}}$ & $1.75 \tilde{o}_{k}$ & $2 \tilde{o}_{k}$ \\
\hline 200 & 3.34 & 4.18 & 5.01 & 5.85 & 6.68 & 1.11 & 1.39 & 1.67 & 1.95 & 2.23 \\
\hline 250 & 2.99 & 3.74 & 4.48 & 5.23 & 5.98 & 0.10 & 1.25 & 1.49 & 1.74 & 1.99 \\
\hline 300 & 2.73 & 3.41 & 4.09 & 4.77 & 5.46 & 0.91 & 1.14 & 1.36 & 1.59 & 1.82 \\
\hline 350 & 2.53 & 3.16 & 3.79 & 4.42 & 5.05 & 0.84 & 1.05 & 1.26 & 1.47 & 1.68 \\
\hline 400 & 2.36 & 2.95 & 3.54 & 4.13 & 4.72 & 0.79 & 0.98 & 1.18 & 1.38 & 1.57 \\
\hline 450 & 2.23 & 2.78 & 3.34 & 3.90 & 4.45 & 0.74 & 0.93 & 1.11 & 1.30 & 1.48 \\
\hline 500 & 2.11 & 2.64 & 3.17 & 3.70 & 4.23 & 0.70 & 0.88 & 1.06 & 1.23 & 1.41 \\
\hline 550 & 2.01 & 2.52 & 3.02 & 3.53 & 4.03 & 0.67 & 0.84 & 1.01 & 1.18 & 1.34 \\
\hline 600 & 1.93 & 2.41 & 2.89 & 3.38 & 3.86 & 0.64 & 0.80 & 0.96 & 1.13 & 1.29 \\
\hline 650 & 1.85 & 2.32 & 2.78 & 3.24 & 3.71 & 0.62 & 0.77 & 0.93 & 1.08 & 1.24 \\
\hline 700 & 1.79 & 2.23 & 2.68 & 3.12 & 3.57 & 0.60 & 0.74 & 0.89 & 1.04 & 1.19 \\
\hline 750 & 1.73 & 2.16 & 2.59 & 3.02 & 3.45 & 0.58 & 0.72 & 0.86 & 1.01 & 1.15 \\
\hline 800 & 1.67 & 2.09 & 2.51 & 2.92 & 3.34 & 0.56 & 0.70 & 0.84 & 0.97 & 1.11 \\
\hline
\end{tabular}

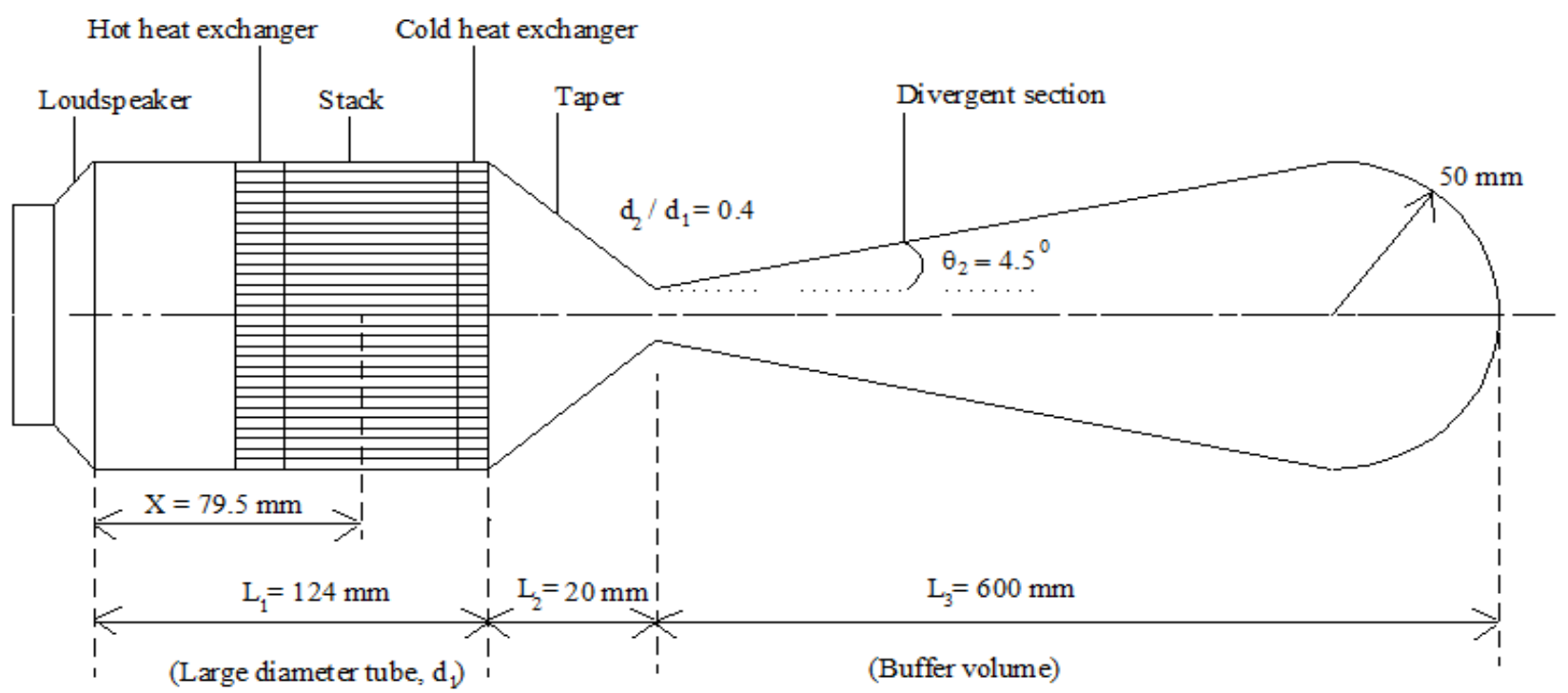

Fig. 3. A $10 \mathrm{~W}$ cooling power TDH resonator design operated with air, and helium gas.

Table 5. Specifications of the TDH resonator design.

$l_{1}=28 \mathrm{~mm}, l_{2}=14 \mathrm{~mm}, l_{3}=75 \mathrm{~mm}, l_{4}=7 \mathrm{~mm}, l_{5}=20 \mathrm{~mm}, l_{6}=550 \mathrm{~mm}, R_{\mathrm{hs}}=50 \mathrm{~mm}, L_{\mathrm{t}}=744 \mathrm{~mm}, 2 y=1 \mathrm{~mm}$,
$2 l=0.557 \mathrm{~mm}, \varepsilon=0.64, d_{1}=3.6 \mathrm{~cm}, d_{2} / d_{1}=0.4, \theta_{2}=4.5^{\circ}, A_{\mathrm{hs}}=0.01571 \mathrm{~m}^{2}, V_{\mathrm{hs}}=2.618 \times 10^{-4} \mathrm{~m}^{3}, A_{\mathrm{t}}=1306.6 \mathrm{~cm}^{2}$,
$V_{\mathrm{t}}=2.08 \mathrm{~L}, P_{\mathrm{v}}=4817.7 \mathrm{~W} / \mathrm{m}^{3}$




\section{Comparative Results Analysis Using DeltaEC: Air versus Helium}

In this section the theoretical performance evaluation of the TDH resonator design (Fig. 3) using DeltaEC software [13] operating with air and helium as working substances are discussed. The DeltaEC is specially designed software for thermoacoustic devices. The DeltaEC is used to predict the theoretical performance of the thermoacoustic model (engine or refrigerator) proposed by the designer before being constructed $[1-7,14]$. The loudspeaker is attached at the left hand side of the resonator system, which provides the required acoustic power input to the resonator system for heat pumping. The electromechanical parameters of the loudspeaker used in this analysis are given in Table 6 . The cross-sectional area of the vibrating diaphragm of the loudspeaker $\left(A_{\mathrm{p}}\right)$ acts as piston for compression and expansion of the gas is given by $10.19 \mathrm{~cm}^{2}$. The electrical resistance $\left(R_{\mathrm{e}}\right)$ and mechanical resistance $\left(R_{\mathrm{m}}\right)$ of the loudspeaker is given by $4 \Omega$ and $3 \mathrm{Nsm}^{-1}$ respectively. The electrical impedance of the loudspeaker $\left(L_{\mathrm{e}}\right)$ is given by 0.0013 $\mathrm{H}$. The mass of the moving part of the loudspeaker system $(\mathrm{m})$ is found to be $9.2 \mathrm{~g}$. The force factor of the moving element $(B l)$ is given by $12.8 \mathrm{~T}-\mathrm{m}$.

Table 6. Electromechanical parameters of the electroacoustic driver

$$
\begin{gathered}
A_{\mathrm{p}}=10.19 \mathrm{~cm}^{2}, R_{\mathrm{e}}=4 \Omega, R_{\mathrm{m}}=3 \mathrm{Nsm}^{-1} 1, L_{\mathrm{e}}=0.0013 \mathrm{H}, \\
m=9.2 \mathrm{~g}, B l=12.8 \mathrm{~T}-\mathrm{m}, \mathrm{s}=0 \mathrm{Nm}^{-1}
\end{gathered}
$$

The spring stiffness $(s)$ for the loudspeaker is a function of the resonator frequency $(f)$ and moving mass $(\mathrm{m})$ [4]. The commercially available loudspeaker with the above specifications has an electro-acoustic conversion efficiency of about $3 \%[4,9]$. Therefore the back volume gas spring system is provided for the commercial loudspeaker (called as driver) to maximize its electro-acoustic efficiency by matching resonator frequency $(f)$ with the driver frequency $\left(f_{\mathrm{d}}\right)$. The resonator system attached with driver has zero spring stiffness $\left(s=0 \mathrm{Nm}^{-1}\right)$. The DeltaEC simulation results as a function of cooling load for the TDH resonator design operating with air, and helium gas as working substances are shown in Figs. 4-12. DeltaEC assumes by default that the resonator and the driver are perfectly insulated with the surrounding. Therefore the hot heat exchanger rejects the electrical power input to the driver and the heat absorbed at the cold heat exchanger.

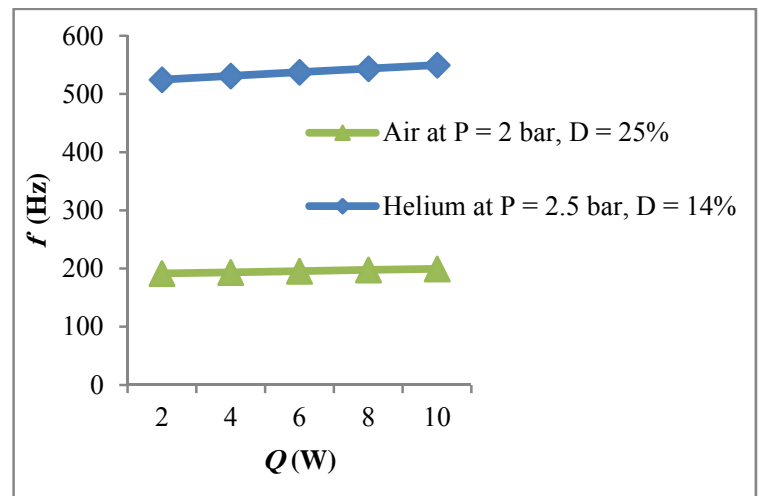

Fig. 4. Operating frequency as a function of cooling load.

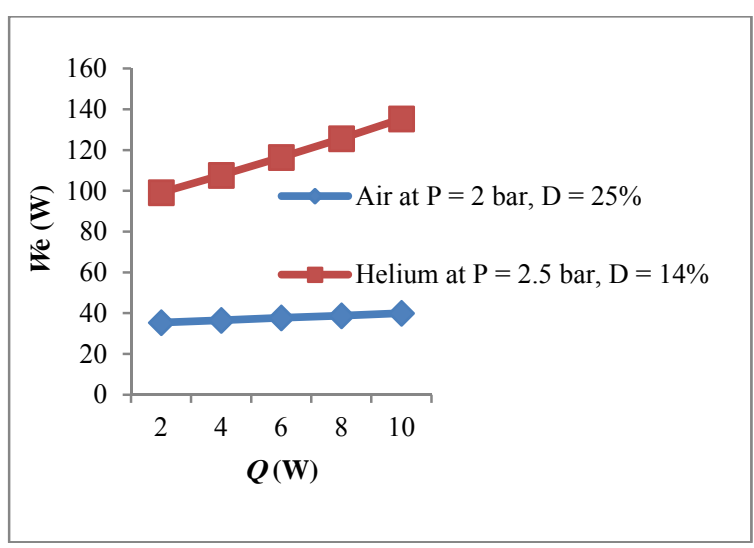

Fig. 5: Electrical power input as a function of cooling load.

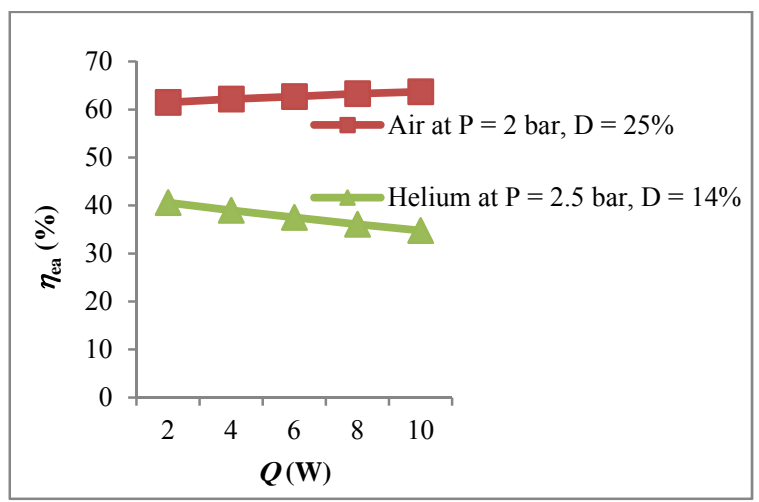

Fig. 6. Electroacoustic efficiency as a function of cooling load.

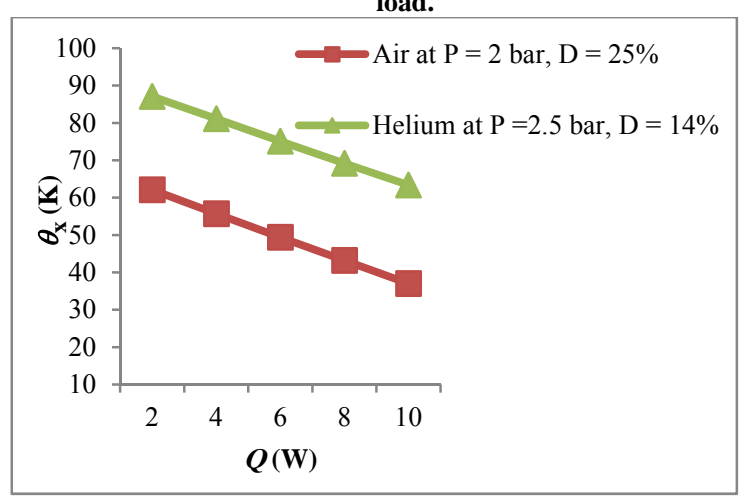

Fig. 7. Temperature gradient across heat exchangers as a function of cooling load.

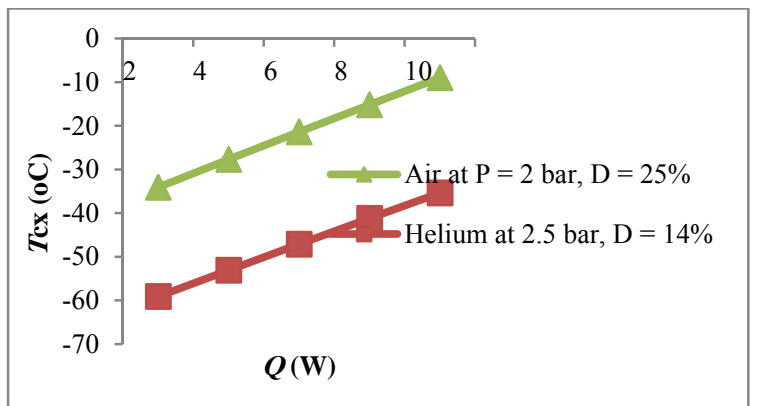

Fig. 8. Cold heat exchanger temperature as a function of cooling load. 


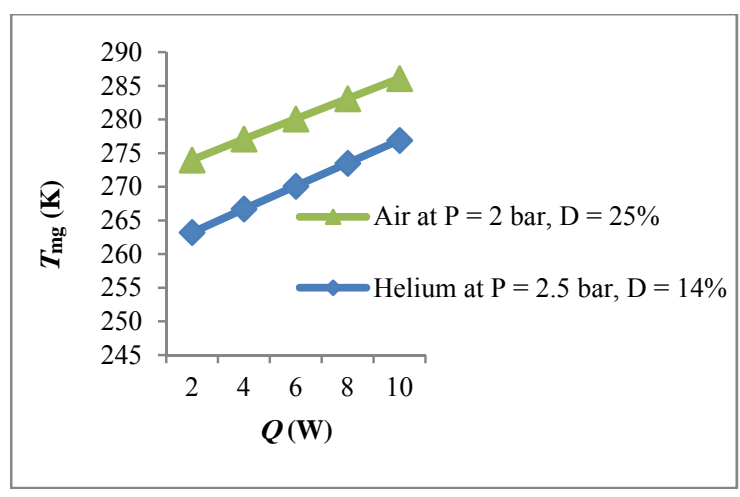

Fig. 9. Mean temperature of gas across heat exchangers as a function of cooling load.

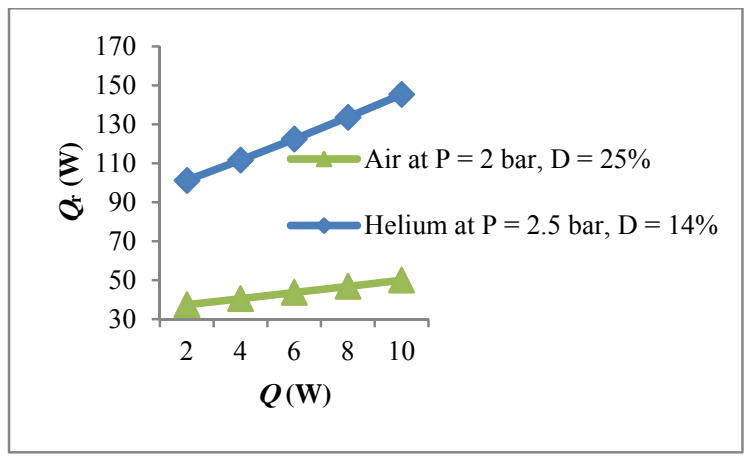

Fig. 10: Heat rejected by the heat exchangers as a function of cooling load.

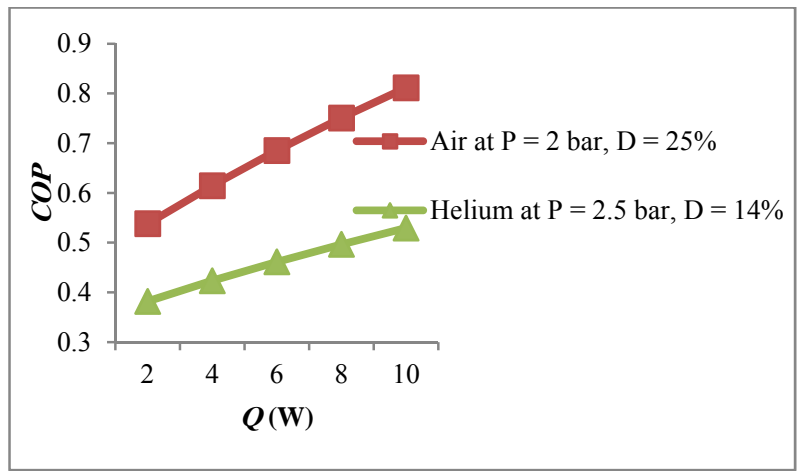

Fig. 11: Coefficient of performance as a function of cooling load.

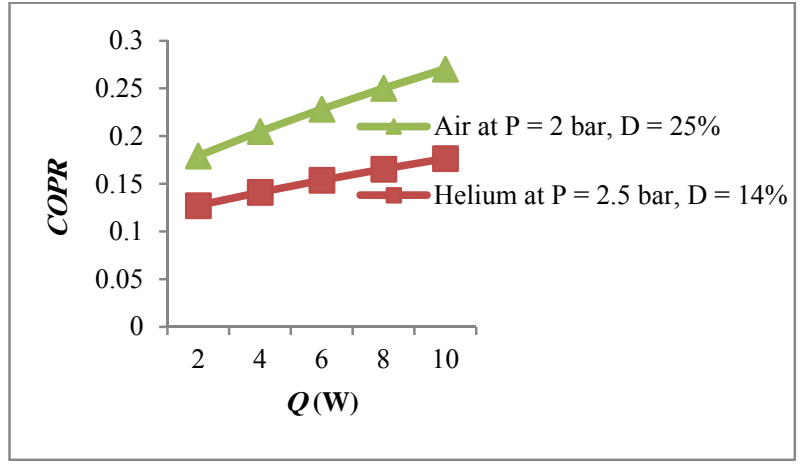

Fig. 12. Coefficient of performance relative to Carnot as a function of cooling load.
Air is operated with a mean pressure $P$ of 2 bar and drive ratio $D 25 \%$, and helium is operated with a mean pressure $P$ of 2.5 bar and drive ratio $14 \%$. The operating frequency $f$ is proportional to the cooling load $Q$ for both the gases. But the helium gas operates at higher frequency compared to air because of higher gas velocity as shown in Fig. 4. The electric power input $W_{\mathrm{e}}$ is proportional to the cooling load for both the gases and helium gas demands more electrical power input compared to air because of low gas density (Fig. 5). The electroacoustic efficiency of the loudspeaker $\eta_{\text {ea }}$ using air as working substance is proportional to cooling load, and is inversely proportional to cooling load for helium gas (Fig. 6). The electroacoustic efficiency of the loudspeaker for air is higher because of the higher density and drive ratio $D(25 \%)$. The temperature gradient across the heat exchangers for both the gases is inversely proportional to the cooling load (Fig. 7). The temperature gradient is higher for helium gas because of higher gas velocity and lower drive ratio $D(14 \%)$. The cold heat exchanger temperature increases with increase in the cooling load for both air and helium gas (Fig. 8). Helium gas shows lower temperature compared to air because of less eddies and turbulence, which reduces nonlinearities in the resonator system. The mean temperature of gas across the hot and cold heat exchangers increases with increase in the cooling load for both the gases (Fig. 9). The mean temperature of gas across the hot and cold heat exchangers for air is higher compared to helium because of higher eddies and turbulence causing nonlinearities in the resonator system at high drive ratio $(25 \%)$. The heat rejected by the hot heat exchanger for both gases is proportional to the cooling load (Fig. 10). The heat rejected by helium gas is higher compared to air because of higher gas velocity and thermal conductivity. The coefficient of performance (COP) and relative COP (COPR) of the refrigerator system is proportional to the cooling load. Helium gas shows lower COP (Fig. 11) and COPR (Fig. 12) at the benefit of lower cold heat exchanger temperature (Fig. 8) compared to air. The COP and COPR are inversely proportional to the cold heat exchanger temperature $T_{\text {cx. }}$

\section{Conclusions}

In this paper, a brief insight into the various optimized resonator designs used by the researchers in the past are discussed, out of which a TDH resonator design is found to be efficient $[1,2]$. A step by step procedure to be followed in designing a laboratory scale thermoacoustic refrigerator model is presented, which can be used as a ready reference for the fresh researchers. Similar to helium gas [1-7], it is also found true for air as working substance such that the thermal and viscous penetration depth decreases with increase in the operating frequency (Table 3 ). And the stack sheet spacing and thickness decreases with increase in operating frequency. Also the stack sheet spacing and thickness increases with increase in the value of half-stack spacing expressed in terms of the thermal penetration depth $\delta_{\mathrm{k}}$ (Table 4). Theoretical performance evaluation of an efficient TDH resonator design operating with air and helium gas as working substance is done using DeltaEC software. The TDH resonator design (Fig. 3) has a surface area $1306.6 \mathrm{~cm}^{2}$ and power density $4817.7 \mathrm{~W} / \mathrm{m}^{3}$, which is in par with the published resonator designs [1-6]. The DeltaEC simulation results for a 10 W TDH resonator design operating with air and helium as working substance shows that air need to be operated at higher drive ratio compared to helium ( $25 \%$ vis-à-vis $14 \%$ ) because of higher gas density. Air operates at lower frequency compared to 
helium (199.5 Hz vis-à-vis $549.7 \mathrm{~Hz})$. Rate of heat rejected at HHX using air is about three times lower compared to helium ( $50 \mathrm{~W}$ vis-à-vis $145.3 \mathrm{~W})$, which causes increase in temperature of the CHX $\left(-9{ }^{\circ} \mathrm{C}\right.$ vis-à-vis $\left.-35.4{ }^{\circ} \mathrm{C}\right)$. Even though air is free and abundantly available in nature, it has higher density causes lower gas velocity and has low thermal conductivity are the detrimental parameters affects $\mathrm{CHX}$ temperature compared to helium. Air has lower temperature difference across the stack $(37 \mathrm{~K}$ vis-à-vis $63.4 \mathrm{~K})$, resulting with an improvement in COP (0.8113 vis-à-vis 0.5294$)$ and COPR (0.2704 vis-à-vis 0.1765$)$ because of decrease in the electrical power input to the driver (Fig. 5). As the COP increases, the CHX temperature also increases, which decreases the refrigeration effect and viceversa. Therefore the working gas is chosen based on the type of cooling application it may be used for.

\section{Acknowledgments}

This work is supported by JSSMVP Mysuru, Principal, HOD (IEM) and all staff of department of Industrial Engineering and Management, JSSATE Bengaluru. I (corresponding author) sincerely thank my guide, co-guide, Prof. G.S.V.L. Narasimham, and Principal and HOD (Mechanical) AIT Chikkamagaluru. Also I thank Bill Ward, John Clark, and Greg Swift, Los Alamos National Laboratory, USA for developing the DeltaEC software and making it freely available for research purpose.

\section{References}

[1] B.G. Prashantha, M.S. Govinde Gowda, S. Seetharamu and G.S.V.L. Narasimham. Resonator Optimization and Studying the Effect of Drive Ratio on Theoretical Performance of a $10-\mathrm{W}$ Cooling Power Thermoacoustic Refrigerator. Int. J. Air-Conditioning \& Refrigeration 2015; 23 (3): 1550020, DOI: $10.1142 / \mathrm{S} 2010132515500200$

[2] B.G. Prashantha, M.S. Govinde Gowda, S. Seetharamu and G.S.V.L. Narasimham. Design and Optimization of a Loudspeaker-Driven $10 \mathrm{~W}$ Cooling Power Thermoacoustic Refrigerator. Int. J. Air-Conditioning and Refrigeration 2014; 22 (3): 1450015, DOI: 10.1142/S201013251450015

[3] B.G. Prashantha, M.S. Govinde Gowda, S. Seetharamu and G.S.V.L. Narasimham. Theoretical Evaluation of 10-W Cooling Power Thermoacoustic Refrigerator. Heat Transfer-Asian Research Journal, Published Online on 3 Oct 2013 , DOI: $10.1002 / \mathrm{htj} .21094$

[4] B.G. Prashantha, M.S. Govinde Gowda, S. Seetharamu and G.S.V.L. Narasimham. Theoretical Evaluation of Loudspeaker for a $10-\mathrm{W}$ Cooling Power Thermoacoustic Refrigerator. Int. J. AirConditioning and Refrigeration 2013; 21 (4): 1350027, DOI: $10.1142 / \mathrm{S} 2010132513500272$
[5] B.G. Prashantha, M.S. Govinde Gowda, and S. Seetharamu. Effect of Mean Operating Pressure on the Performance of Stack-Based Thermoacoustic Refrigerator. Int. J. Thermal and Environmental Engineering $2013 ; \quad 5 \quad$ (1):83-89, DOI: 10.5383/ijtee.05.01.009

[6] B.G. Prashantha, M.S. Govinde Gowda, S. Seetharamu and G.S.V.L. Narasimham. Design and Analysis of Thermoacoustic Refrigerator. Int. J. Air-Conditioning and Refrigeration 2013; 21 (1): $1350001 \quad$ (10 pages), DOI: $10.1142 / \mathrm{S} 2010132513500016$

[7] B.G. Prashantha, M.S. Govinde Gowda, S. Seetharamu and G.S.V.L. Narasimham. Stack Optimization of Thermoacoustic Refrigerator. The J. CPRI, Bangalore 2012; 8 (1):97-102, ISSN 0973 0338.

[8] G.W. Swift. Thermoacoustic Engines. J. Acoust. Soc. Am. 1988; 84 (4):1145-1180.

[9] M.E.H. Tijani. Loudspeaker-Driven ThermoAcoustic Refrigeration. Ph.D. Thesis, Eindhoven University of Technology; 2001.

[10] B.G. Prashantha and M.S. Govinde Gowda. A Review of Literature on Thermoacoustic Refrigerator. National Conference on Advances in Mechanical Engineering, MIT, Manipal, India, 2011.

[11] B.G. Prashantha, M.S. Govinde Gowda and S. Seetharamu. A Study on Thermoacoustic Refrigerator. National Conference on Emerging Trends in Mechanical Engineering and Sciences, Bannari Amman institute of Technology, Sathyamangalam, Tamil Nadu, India, 2007.

[12] B.G. Prashantha and M.S. Govinde Gowda. Standing and Travelling Wave Thermoacoustic Refrigerator. Int. Conference on Energy Engineering and Eco-Balance, Mulshi institute of Technology and Research, Pune, Maharashtra, India, 2007.

[13] B. Ward, J. Clark and G.W. Swift. Design Environment for Low-Amplitude Thermoacoustic Energy Conversion (DeltaEC software). Version 6.2, Los Alamos National Laboratory, 2008, Available at http://www.lanl.gov/thermoacoustics.

[14] B.G. Prashantha and M.S. Govinde Gowda. Design of Thermoacoustic Refrigerator Using DeltaEC. Int. Conference on Frontiers in Mechanical Engineering, NITK Surathkal, 2010. 